\title{
Sex, Pramipexole and Tiagabine Affect Behavioral and Hormonal Response to Traumatic Stress in a Mouse Model of PTSD
}

OPEN ACCESS

Edited by:

Claudio Bucolo,

University of Catania, Italy

Reviewed by:

Gian Marco Leggio,

University of Catania, Italy

Hyo Young Lee,

Dongseo University, South Korea

${ }^{*}$ Correspondence: Natalia Malikowska-Racia malikow@if-pan.krakow.pl

Specialty section: This article was submitted to

Neuropharmacology,

a section of the journal

Frontiers in Pharmacology

Received: 16 April 2021

Accepted: 19 May 2021

Published: 30 June 2021

Citation:

Malikowska-Racia N, Salat $K$, Gdula-Argasinska J and Popik P

(2021) Sex, Pramipexole and

Tiagabine Affect Behavioral and Hormonal Response to Traumatic Stress in a Mouse Model of PTSD.

Front. Pharmacol. 12:691598. doi: 10.3389/fphar.2021.691598

\begin{abstract}
Natalia Malikowska-Racia ${ }^{1,2 *}$, Kinga Salat ${ }^{2}$, Joanna Gdula-Argasinska ${ }^{3}$ and Piotr Popik ${ }^{1,4}$
${ }^{1}$ Department of Behavioral Neuroscience and Drug Development, Maj Institute of Pharmacology, Polish Academy of Sciences, Krakow, Poland, ${ }^{2}$ Department of Pharmacodynamics, Faculty of Pharmacy, Jagiellonian University Medical College, Krakow, Poland, ${ }^{3}$ Department of Radioligands, Faculty of Pharmacy, Jagiellonian University Medical College, Krakow, Poland, ${ }^{4}$ Faculty of Health Sciences, Jagiellonian University Medical College, Krakow, Poland
\end{abstract}

Posttraumatic stress disorder (PTSD) has been associated with abnormal regulation of the hypothalamic-pituitary-adrenal gland axis (HPA). Women demonstrate a more robust HPA response and are twice as likely to develop PTSD than men. The role of sex hormones in PTSD remains unclear. We investigated whether post-trauma chronic treatment with the GABA-ergic agent tiagabine and dopamine-mimetic pramipexole affected the behavioral outcome and plasma levels of corticosterone, testosterone, or $17 \beta$-estradiol in female and male mice. These medications were investigated due to their potential capacity to restore GABA-ergic and dopaminergic deficits in PTSD. Animals were exposed to a single prolonged stress procedure (mSPS). Following 13 days treatment with tiagabine $(10 \mathrm{mg} / \mathrm{kg})$ or pramipexole $(1 \mathrm{mg} / \mathrm{kg})$ once daily, the PTSD-like phenotype was examined in the fear conditioning paradigm. Plasma hormones were measured almost immediately following the conditioned fear assessment. We report that the exposure to mSPS equally enhanced conditioned fear in both sexes. However, while males demonstrated decreased plasma corticosterone, its increase was observed in females. Trauma elevated plasma testosterone in both sexes, but it had no significant effects on $17 \beta$-estradiol. Behavioral manifestation of trauma was reduced by pramipexole in both sexes and by tiagabine in females only. While neither compound affected corticosterone in stressed animals, testosterone levels were further enhanced by tiagabine in females. This study shows sex-dependent efficacy of tiagabine but not pramipexole in a mouse model of PTSD-like symptoms and a failure of steroid hormones' levels to predict PTSD treatment efficacy.

Keywords: Pramipexole, tiagabine, corticosterone, PTSD, trauma, sex, HPA

\section{INTRODUCTION}

Traumatic stress triggers a maladaptive response of the hypothalamus-pituitary-adrenal gland axis (HPA), evidenced as abnormally fast negative feedback (Yehuda et al., 1993) that is one of the core hallmarks of posttraumatic stress disorder (PTSD). However, the mechanism by which HPA adaptability contributes to PTSD has not yet been fully explained; for a review, see Dunlop and Wong (2019). The twice higher prevalence of PTSD in women (Kessler et al., 1995; Yehuda et al., 
2015) coincides with sex-dependent HPA responsiveness (Heck and Handa, 2019). Considering that sex affects the response to stress and thus PTSD susceptibility, we hypothesized that it might also affect PTSD's pharmacotherapy efficacy.

To address this issue in an animal model of PTSD, we used the mouse single prolonged stress (mSPS) procedure, which resembles human exposure to traumatic stress (Perrine et al., 2016). Trauma exposure predominantly affects fear conditioning, which manifests as an exaggerated fear reaction that tends to generalize, and fails to extinguish (Orr et al., 2000; Morey et al., 2015). The homology of conditioned fear between non-human mammals and humans is striking and constitutes face and construct validities of the mSPS and SPS models (VanElzakker et al., 2014; Perrine et al., 2016; Verbitsky et al., 2020).

Here we examined the effects of pramipexole, an antiparkinsonian $\mathrm{D}_{2}$-like receptor agonist, which reduced conditioned freezing and decreased immobility in male mice exposed to mSPS (Malikowska-Racia et al., 2019a). Pramipexole may improve trauma memory extinction (Gerlicher et al., 2018). Furthermore, as the $\mathrm{D}_{3}$-preferring agonist, pramipexole aims at the promising target for the treatment of neuropsychiatric disorders, which share some common symptoms with PTSD (Leggio et al., 2019; Leggio et al., 2021; for the review, see; Kiss et al., 2021). The concept of $\mathrm{D}_{3}$ receptors' role in PTSD has already been proposed, being further discussed in Torrisi et al. (2019). Nevertheless, the rationale of using pramipexole in this study primarily relies on the hypothesis that women and men trauma victims present different, dopamine-dependent adaptation to stress. Increased dopamine levels following stress have been shown to prevent abnormal HPA response and promote adaptive coping strategies; for the review, see Cabib and Puglisi-Allegra (2012). This mechanism is sex-dependent as following an uncontrollable stressor, female rats display prolonged passive coping and greater VTA dopamine decline than males (Rincón-Cortés and Grace, 2017). Thus, considering that dopamine-driven stress adaptation in females is not as effective as in males, we hypothesized that dopamine-mimetic medications, such as pramipexole, would demonstrate higher efficacy in traumatized female mice.

Another compound investigated here was the GABA-mimetic anticonvulsant medication tiagabine, which in our earlier report (Malikowska-Racia et al., 2019b) decreased immobility and anxiety following acute administration in mSPS-exposed male mice. Of note, GABA-ergic deficits have been demonstrated in PTSD patients (Geuze et al., 2008; Trousselard et al., 2016; Rasmusson et al., 2019), most likely resulting from an insufficient neurosteroid modulation of GABA-A receptors. Since impaired neurosteroidogenesis as found in PTSD females further attenuates GABA-ergic tone (Pineles et al., 2018; Rasmusson et al., 2019), we hypothesized that the efficacy of tiagabine, which inhibits GABA reuptake, would be greater in female mice. This is because the positive modulation of GABA-A receptors conferring neurosteroid sensitivity is fundamental for tonic HPA inhibition (Sarkar et al., 2011).

To closely model the clinical setting, we treated mice almost immediately after trauma. It has been shown that there is a "golden hours" time window in humans exposed to trauma, during which preventive treatment should be initiated (Carmi et al., 2016). Mice were treated with tiagabine or pramipexole for the following two weeks, i.e., up to the time they showed PTSDlike symptoms. Following sub-chronic treatment and behavioral tests, we examined the levels of plasma corticosterone as well as testosterone and $17 \beta$-estradiol because sex steroids have been shown to modify HPA response (Heck and Handa, 2019).

\section{MATERIALS AND METHODS}

\section{Chemical Compounds Used in the Study}

Pramipexole dihydrochloride $(1 \mathrm{mg} / \mathrm{kg}$, s.c.; Sigma Aldrich, Poland) and tiagabine $(10 \mathrm{mg} / \mathrm{kg}$, i.p., Tocris Bioscience, Germany) were dissolved in $0.9 \%$ saline or suspended in $1 \%$ Tween 80 , respectively. Both medications were injected in a volume of $10 \mathrm{ml} / \mathrm{kg}$ once daily at 8:00-9:00 AM for 13 days. Doses were selected based on our previous studies (Salat et al., 2015; Malikowska-Racia et al., 2019a). Control groups received $0.9 \%$ saline solution $(10 \mathrm{ml} / \mathrm{kg})$. Isoflurane, used for anesthesia in the mSPS procedure, was provided by Baxter (Puerto Rico, United States).

\section{Ethics, Animals, and Housing Conditions}

All procedures were approved by the $1^{\text {st }}$ Local Ethics Committee of the Jagiellonian University in Kraków (292/2019) and were in full accordance with ethical standards laid down in respective Polish and EU regulations (Directive No. 86/609/EEC). Subjects were 48 male and 48 female albino Swiss CD-1 5-week old mice, weighing $18-22 \mathrm{~g}$ at the arrival to the laboratory. The animals were purchased from the Animal Breeding Farm of the Jagiellonian University Faculty of Pharmacy.

The animals were kept in groups of eight in standard plastic cages $(42 \times 26.5 \times 18 \mathrm{~cm})$ and were housed under controlled conditions (room temperature of $22 \pm 2{ }^{\circ} \mathrm{C}$, light/dark (12:12) cycle, lights on at 7:00 AM, humidity $50-60 \%$ and free access to food and water). For the tests, the animals were selected randomly. All experiments were performed between 8:00 AM and 3:00 PM in a sound-attenuated room under dim light and continuous white noise of $65 \mathrm{~dB}$.

\section{Experimental Schedule}

Mice were exposed to $\mathrm{mSPS}$ or sham mSPS procedure at their age of 6 weeks. Twenty-four $h$ following trauma, the treatment with pramipexole, tiagabine or saline began. Following 13 days administration, on days 14-15, the fear conditioning test was performed. Afterward, mice were decapitated, and blood samples were collected to determine corticosterone, testosterone, and $17 \beta$ estradiol plasma levels. The study timeline is presented in Figure 1.

\section{Behavioral Procedures}

\section{Mouse Single Prolonged Stress Procedure}

Mouse mSPS was done as in Perrine et al. (2016) with some modifications (Malikowska-Racia et al., 2019a). Briefly, mice were exposed to a series of four different stressors: the tight 


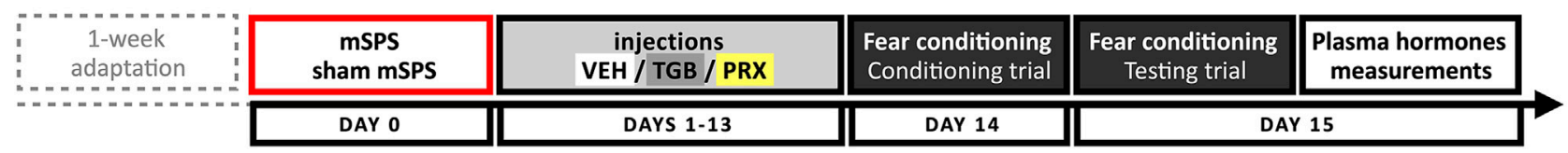

FIGURE 1 | Experimental schedule. Male and female mice were exposed to mSPS ( $N=24+24)$ or sham mSPS ( $N=24+24)$. Twenty-four hours following trauma, 13-days long treatment with tiagabine (TGB, $10 \mathrm{mg} / \mathrm{kg}$, i.p.) pramipexole (PRX, $1 \mathrm{mg} / \mathrm{kg}$, s.c.) or saline began. On days 14-15, conditioned fear was examined, and $1 \mathrm{~h}$ later, the animals were decapitated, and blood samples were collected for further evaluation of plasma corticosterone, testosterone, and 173 -estradiol (for each hormone and sex, $N=8$ ). Abbreviations: mSPS - mouse single prolonged stress, PRX - pramipexole, TGB - tiagabine.

restrainment $(2 \mathrm{~h})$, water forced group swim $(10 \mathrm{~min})$ and the exposure to a rat predator scent $(15 \mathrm{~min})$. Due to the current ethical requirements, the original diethyl ether anesthesia (Perrine et al., 2016) has been replaced with isoflurane anesthesia lasting for 3-4 min. Control mice underwent a sham-mSPS procedure, which consisted of handling and weighing. Next, all mice were left in the home cages for an undisturbed period of $24 \mathrm{~h}$.

\section{Contextual Fear Conditioning}

Contextual fear conditioning was performed as reported (Malikowska-Racia et al., 2019a), except for an auditory cue's absence. We used a white-painted cage (Bioseb, France; $25 \times 18 \times$ $16 \mathrm{~cm}$ ), floored with metal plates that delivered electroshocks. On the first day, each mouse was individually placed in the cage for a $1 \mathrm{~min}$ habituation period. During the next $2 \mathrm{~min}$, each animal was exposed to five electric shocks (duration: $2 \mathrm{~s}$, current intensity: $0.8 \mathrm{~mA}$ ). The next day mice were placed individually in the same cage again, and after a $30 \mathrm{~s}$ habituation period, the 2 min testing period started. The dependent measure, i.e., the freezing response time defined as the total immobility excluding movements enabling respiration, was recorded and then scored manually by a trained observer blind to the treatment conditions.

\section{Measurement of Steroid Hormones}

Animals were decapitated between 10:00 AM and 12:00 AM, and trunk blood was collected and centrifuged $\left(2000 \times \mathrm{g}\right.$, at $4^{\circ} \mathrm{C}$ for $10 \mathrm{~min}$ ) to collect plasma. Levels of corticosterone, testosterone, and $17 \beta$-estradiol were measured using commercially available ELISA kits (Neogen Corp., Lexington, United States) following the manufacturer's instructions. The samples and the standards were run in duplicates. We used three types of 96-well plates (corticosterone \#4028110; testosterone \#402510, 17 $\beta$-estradiol \#402110). The horseradish peroxidase was added to the antibody-coated plate and incubated for $1 \mathrm{~h}$ at room temperature $\left(\sim 24^{\circ} \mathrm{C}\right)$. Following incubation, the plate was washed three times with a wash buffer provided with the kit. Next, the mixture of tetramethylbenzidine and $\mathrm{H}_{2} \mathrm{O}_{2}$ was added and incubated for $30 \mathrm{~min}$ at room temperature. The bound conjugate was detected using a plate reader (POLARstar Omega, BMG LABTECH, Germany) at an absorbance of $650 \mathrm{~nm}$. Data were quantified based on the standard calibration curves.

\section{Statistical Analysis}

For statistical analysis, the IBM SPSS Statistics (v.24, NY, United States) was used.
Using 3-way ANOVA, we first investigated the effects of sex, treatment, and mSPS as well as whether sex affected treatment efficacy, whether sex affected mSPS effects, and whether treatment affected mSPS response. The sex $\mathrm{x}$ treatment $\mathrm{x}$ mSPS interaction was examined to determine whether treatment efficacy was similar in both sexes and depended on mSPS.

Since the sex $\mathrm{x}$ treatment $\mathrm{x}$ mSPS interaction was not significant for fear conditioning and estradiol levels' data (Figures 2A,B, Figures 2E,F, respectively), and because testosterone levels were $20 \mathrm{x}$ higher in males than females (Figures 2G,H), these data sets were then subjected to two independent 2-way ANOVAs for each sex. It served to examine whether mSPS and/or treatment affected the given phenotype and whether the effects of mSPS depended on the treatment. The two a priori questions: 1) whether mSPS affected the dependent variable in vehicle-controls and, 2) whether treatment with tiagabine or pramipexole exerted different effects than the respective vehicle controls, were assessed with contrast analyses (Cardinal and Aitken, 2006).

A linear regression model and correlation analyses were used to determine $r, R^{2}$ and statistical significance of the relationship between freezing time duration and plasma hormones levels.

The detailed statistics are presented in the Supplementary Material.

Sample sizes were estimated with the use of $\mathrm{G}^{\star}$ Power Software (v. 3.1.9.4.) (Perugini et al., 2018), which indicated minimal sample size $N=5$ and actual power of 0.8 for ANOVAs (Cohen's $f=0.45$; $a=0.05$ ) or $N=4$ and actual power of 0.83 for contrast analyses (Cohen's $d=2.5$; $\alpha=0.05$ ).

\section{RESULTS}

\section{Effects of mSPS, Pramipexole and Tiagabine on Conditioned Fear}

Since 3-way ANOVA showed no significant sex $\mathrm{x}$ treatment $\mathrm{x}$ mSPS interaction, data were analyzed with two 2-way ANOVAs. Both females (Figure 2A) and males (Figure 2B) exposed to mSPS presented prolonged freezing [mSPS effect for females: $\mathrm{F}(1,42)=34.184$ and males: $\mathrm{F}(1,42)=31.309, p<0.001]$. Treatment affected freezing in both sexes $[\mathrm{F}(2,42)=16.41, p<$ 0.001 and $F(2,42)=4.52, p<0.05$ for females and males, respectively], and treatment efficacy depended on stress exposure in females but not males [mSPS $\mathrm{x}$ treatment: $\mathrm{F}(2,42)=$ 4.787, $p<0.05$ and $\mathrm{F}(2,42)=2.418$, NS, respectively].

Contrast analyses revealed that mSPS increased freezing both in females $[t(42)=5.656, p<0.001]$ and males: $[t(42)=4.012$, 


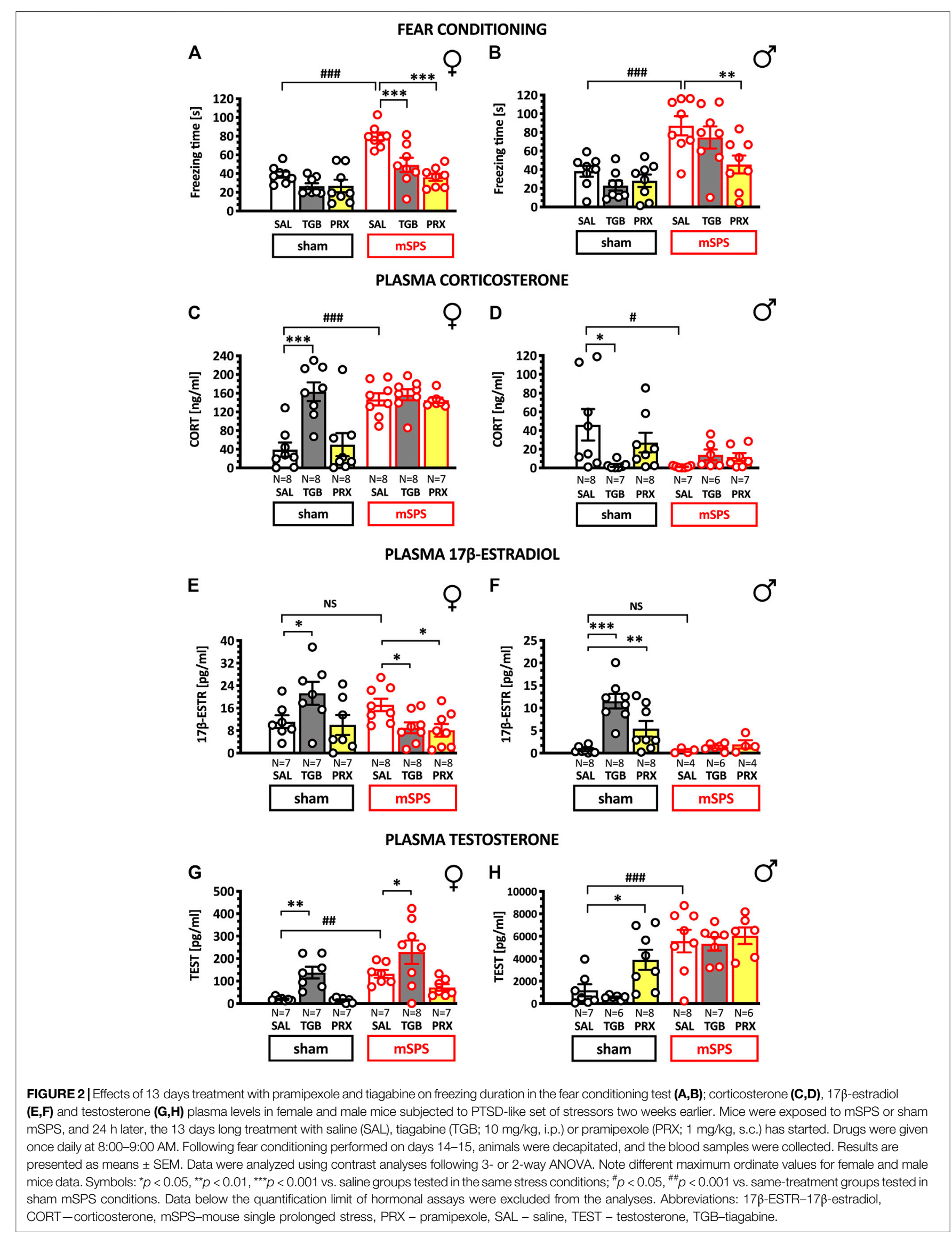


$p<0.001]$. In stressed females both tiagabine $[t(42)=4.221]$ and pramipexole $[t(42)=6.048, p<0.001]$ reduced conditioned freezing. In stressed males, only pramipexole reduced conditioned freezing $[t(42)=3.411, p<0.01]$. In the sham conditions, none of the medications affected the freezing response (Figures 2A,B).

These data suggest that mSPS applied two weeks earlier increased conditioned freezing and that pramipexole effectively reduced it in both sexes, while tiagabine reduced it in females only.

Effects of mSPS, pramipexole and tiagabine on plasma corticosterone level.

Figures 2C,D demonstrate that mSPS exposure $[\mathrm{F}(1,78)=$ 9.33, $p<0.01]$, sex $[\mathrm{F}(1,78)=154, p<0.001]$ and treatment $[\mathrm{F}(2,78)=4.5, p<0.05]$ affected plasma corticosterone as revealed by 3-way ANOVA. The sex $\mathrm{x}$ treatment $\mathrm{x}$ mSPS interaction was also significant $[\mathrm{F}(2,78)=10.03, p<0.001]$, suggesting complex inter-relation among these factors.

Sex modified the long-term effects of $\operatorname{mSPS}[\mathrm{F}(1,78)=25.96, p<$ $0.001]$ and contrast analyses revealed corticosterone increase in stressed females as compared to sham-mSPS females $[t(78)=5.672, p<0.001$; Figure 2C]. In males, trauma exerted an opposite effect in that a decrease of corticosterone levels was observed in stressed- $[t(78)=2.29$, $p<0.05]$ as compared to sham-mSPS subjects; Figure 2D.

The treatment produced different effects on plasma corticosterone in males and females [treatment $\mathrm{x}$ sex $\mathrm{F}(2,78)=$ 10.34, $p<0.001]$. Specifically, due to tiagabine, corticosterone levels were higher in non-stressed females $[t(78)=6.529, p<$ $0.001]$ and lower in non-stressed males $[t(78)=2.208, p<0.05]$ as compared with respective saline controls. However, either tiagabine or pramipexole failed to affect corticosterone levels in traumatized mice.

These data suggest that mSPS increased and decreased corticosterone in females and males respectively. Neither tiagabine nor pramipexole affected corticosterone response in traumatized animals. In sham mice, however, tiagabine increased corticosterone in females and decreased it in males. The effects of tiagabine somewhat resemble the effects of trauma because mSPS per $\underline{s e}$ also increased corticosterone in females and decreased it in males.

\section{Effects of mSPS, Pramipexole and Tiagabine on Plasma Estradiol Level}

While 3-way ANOVA demonstrated significant effects of sex, mSPS and treatment on estradiol plasma levels, it also revealed insignificant $\mathrm{mSPS} x$ treatment $\mathrm{x}$ sex interaction. Thus, data were further analyzed with two separate 2-way ANOVAs in females and males. These analyses revealed significant treatment $\mathrm{x}$ stress interaction in females $[\mathrm{F}(2,39)=5.539, p<0.01$ and males $\mathrm{F}(2,32)$ $=7.67, p<0.01]$, suggesting that the treatment effect depended on prior stress exposure. Nonetheless, neither in females (Figure 2E) nor in males (Figure 2F), prior exposure to mSPS affected plasma estradiol levels, suggesting that this hormonal phenotype might not be associated with PTSD-like symptomatology.

Of note, in non-stressed females $[t(39)=2.513 ; p<0.05]$ and males $[t(32)=6.724 ; p<0.001]$ tiagabine increased plasma estradiol levels. In contrast, in mSPS exposed females but not males, both tiagabine $[t(39)=2.17 ; p<0.05]$ and pramipexole $[t(39)=2.4 ; p<0.05]$ reduced this hormone level. This may suggest that in females, reduced estradiol levels could be associated with the treatment efficacy. Tiagabine-induced estradiol increase in non-stressed females (Figure 2E) resembling a similar corticosterone increase (see Figure 2C).

Overall, these data suggest that plasma estradiol levels are not indicative of the prior severe stress in mice in the present laboratory conditions. Because tiagabine increased plasma estradiol levels in non-stressed mice of both sexes and decreased this hormone level in stressed females, one may conclude that the effects of tiagabine depended on prior stress exposure.

\section{Effects of mSPS, Pramipexole and Tiagabine on Plasma Testosterone Level}

Because testosterone levels were, as expected, much higher in males, data were a priori analyzed with separate 2-way ANOVAs in males and females. These analyses revealed that treatment efficacy did not depend on prior stress exposure in females $[\mathrm{F}(2,37)=0.749]$ and in males $[\mathrm{F}(2,36)=1.681]$. Nonetheless, contrast analyses revealed that in females $[t(37)=2.869 ; p<0.01$; Figure 2G] and in males $[t(36)=4.213 ; p<0.001$; Figure $2 \mathbf{H}]$ the prior exposure to mSPS increased testosterone levels.

Neither treatment with pramipexole nor tiagabine normalized an increased testosterone due to mSPS in male mice (Figure $\mathbf{2 H}$ ). Pramipexole elevated testosterone in non-stressed males $[t(36)=$ 2.609; $p<0.05$ ] and had no effect in females (Figure 2G). Tiagabine increased female testosterone in the sham $[t(37)=3.003 ; p<0.01]$ and mSPS-conditions $[t(37)=2.524 ; p<0.05$; Figure 2G].

Altogether, mSPS increased plasma testosterone in both sexes, and tiagabine increased testosterone in stressed and sham females, while pramipexole did so only in sham-stressed males.

\section{Associations Between Conditioned Freezing Response and Plasma Steroids An Association Between Freezing Response and Plasma Corticosterone Levels}

Figure 3A demonstrates the positive $\left(r==0.67 ; R^{2}==0.45 ; \mathrm{F}\right.$ $[1,14]==11.8 ; p \mathrm{P}<0.01)$ correlation between freezing response and plasma corticosterone levels in female mice. Figure 3B demonstrates negative $\left[r=-0.69 ; R^{2}=0.48, \mathrm{~F}(1,13)=12.47\right.$; $p<0.01]$ correlation between freezing response and plasma corticosterone levels in male mice. A similar analysis disregarding the sex of mice demonstrated no correlation between freezing and corticosterone levels (Figure 3C).

\section{An Association Between Freezing Response and Plasma Estradiol Levels}

There was no significant correlation between freezing response and plasma estradiol levels in female and male mice. For data and statistical analysis, see Supplementary Material.

\section{An Association Between Freezing Response and Plasma Testosterone Levels}

Figures 3D,E demonstrate significant positive correlation between freezing response and plasma testosterone levels in female $\left[r=0.78 ; R^{2}=0.61 ; \mathrm{F}(1,12)=19.02 ; p<0.001\right]$ and 


\section{CONDITIONED FREEZING \& PLASMA CORTICOSTERONE}

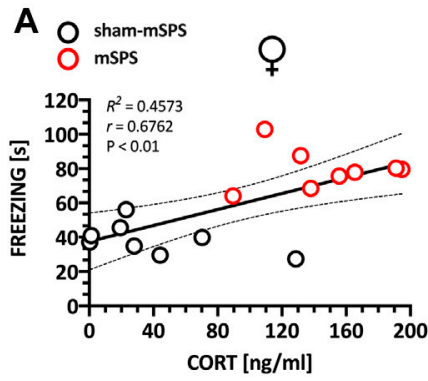

B

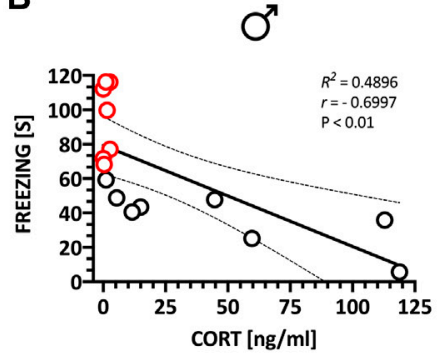

C

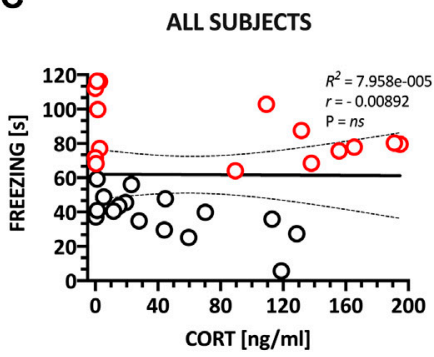

CONDITIONED FREEZING \& PLASMA TESTOSTERONE
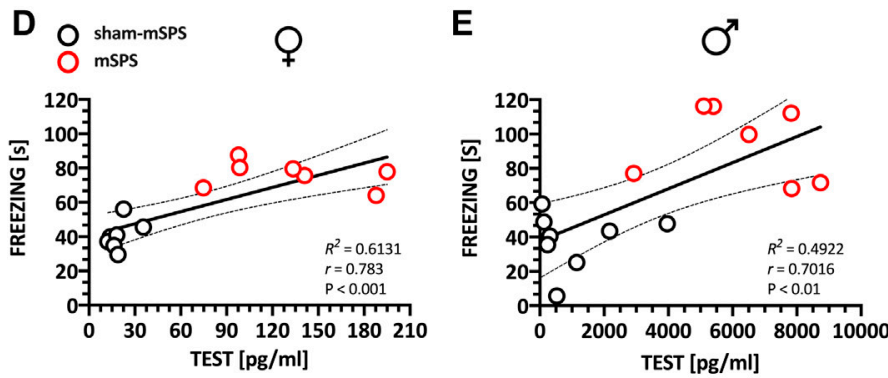

F

ALL SUBJECTS

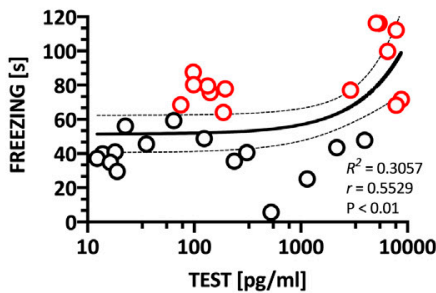

FIGURE 3 | Correlations between behavioral and hormonal responses to trauma in female and male drug-naive mice, two weeks following mSPS. The duration of conditioned freezing was positively and negatively correlated with the plasma levels of corticosterone in female (A) and male mice (B), respectively. No significant correlation was observed if the sex factor was collapsed (C). Conditioned freezing was positively correlated with plasma testosterone in mice of both sexes (D-F). Regression analyses were used to determine $R^{2}$ and statistical significance. Correlated data sets were already presented as sham and mSPS controls treated with saline in the source Figures 2A,B for conditioned freezing, Figures 2C,D for plasma corticosterone and Figures 2G,H for plasma testosterone. For $17 \beta$-estradiol data, see Supplementary Materials. Abbreviations: $17 \beta$-ESTR-17 $\beta$-estradiol, CORT-corticosterone, mSPS-mouse single prolonged stress, TEST-testosterone.

male $\left[r=0.7 ; R^{2}=0.4922 ; \mathrm{F}(1,13)=2.92 ; p<0.01\right]$ mice. A similar analysis disregarding the sex of mice also demonstrated positive correlation between freezing and testosterone levels [Figure 3F, $\left.r=0.55 ; R^{2}=0.3057 ; \mathrm{F}(1,27)=11.89 ; p<0.01\right]$.

\section{DISCUSSION}

This study examined whether sex affected behavioral and hormonal responses in a mouse model of PTSD-like symptoms and whether treatment with tiagabine or pramipexole could affect stress-induced phenotypes. While both females and males showed increased conditioned fear due to the trauma, hormonal responses were far more complex.

\section{Severe Stress Affects Behavior and Hormone Levels in Mice}

Sex Differences in the Behavioral Response to Trauma Earlier research, done chiefly in male rodents, demonstrate prolonged conditioned freezing several days following SPS (e.g., Keller et al., 2015; Malikowska-Racia et al., 2019a; for review, see; Verbitsky et al., 2020) and well correspond with the exaggerated fear response in PTSD patients as defined by DSM-5 (American Psychiatric Association, 2013). The twice higher prevalence of PTSD in women (Kessler et al., 1995; Yehuda et al., 2015) has been addressed in the SPS rat, but not mice studies. In this respect, traumatized female rats display more avoidance (Nahvi et al., 2019) and anhedonia-like behavior as well as social interaction impairments (Pooley et al., 2018), suggesting a stronger depressive-like phenotype. However, traumatized rat females are less than males prone to develop exaggerated conditioned fear (Keller et al., 2015). This corresponds with the Clark et al. (2019) findings from mice showing that also nontraumatized females manifest less robust though more persistent conditioned freezing than males.

Our mice's results appear not to support the latter observations because we report prolonged conditioned freezing in both sexes. This discrepancy could be due to the species differences in response to trauma, the fact that we used isoflurane rather than ether anesthesia, some technical differences in the conditioned fear protocol, and/or other not identified factors. Nevertheless, from the fact that we observed increased conditioned freezing in both sexes (Figures $\mathbf{2 A , B}$ ), one may conclude that the present experimental conditions fulfilled construct and face validities of mSPS as a PTSD model. Clearly, more research is needed to determine the optimal conditions for post-SPS/mSPS testing to reflect sexdependent PTSD susceptibility in humans accurately.

\section{Hormonal Sex Differences}

\section{Sex-dependent Effect on Plasma Corticosterone Level}

Exposure to mSPS increased plasma corticosterone in females while reduced it in males (Figures 2A,B). Because both sexes 
showed trauma-induced prolonged conditioned freezing, corticosterone plasma levels were positively correlated with freezing duration in females and negatively correlated in males.

The release of cortisol in PTSD patients is abnormally reduced by HPA fast negative feedback (Yehuda et al., 1993; Yehuda et al., 2015), resulting in low cortisol that frequently correlates with the PTSD symptoms exacerbation (Aardal-Eriksson et al., 2001). Present report and other preclinical data on male mice agree with human findings (Liberzon et al., 1997; Kohda et al., 2007; Perrine et al., 2016; Pooley et al., 2018). The negative correlation between plasma corticosterone levels and conditioned freezing (Dopfel et al., 2019), extinction deficits, and other manifestations of PTSD-like behaviors (Danan et al., 2018) have already been reported in traumatized male rats.

Nevertheless, the simple causality of low cortisol and PTSD symptoms has already been challenged, both in rodent studies (e.g., Sillivan et al., 2017; Torrisi et al., 2021) and in the clinic. Reports on unchanged or even elevated cortisol in PTSD patients had prompted further research suggesting that low cortisol is associated with trauma exposure or symptoms severity (AardalEriksson et al., 2001; Morris et al., 2016; Josephs et al., 2017; Speer et al., 2019). Beyond experimental heterogeneity that contributes to the contradictory reports (Speer et al., 2019), earlier research noted that lower cortisol had been more consistently reported in male than female subjects (Gill et al., 2005; Metzger et al., 2008). It agrees with our findings (Figures $\mathbf{3 A - C}$ ) and suggests that studies disrespecting sex variable may bias final conclusions.

The pivotal role of sex in hormonal response to trauma is supported by an earlier rodent study by Pooley et al. (2018). These authors observed enhanced HPA control and low plasma corticosterone in traumatized male rats as well as reduced HPA negative feedback and higher corticosterone in females. The enhanced corticosterone response of traumatized female rodents resembles HPA hyposensitivity in depressive disorder and a "female"-specific PTSD-like phenotype (Pooley et al., 2018). While PTSD-suffering and depressed males display opposite HPA regulation manifesting as enhanced and decreased circulating glucocorticoids, females present HPA hyposensitivity and thus increased glucocorticoids in depression and PTSD (Yehuda, 2002; Kessler, 2003).

\section{Effects on Plasma Testosterone and 17 $\beta$-Estradiol}

While stress exposure did not affect estradiol levels, testosterone increased in both sexes following trauma and its levels were positively correlated with the conditioned freezing. This agrees with Karlović et al. (2012), who showed increased testosterone levels in soldiers suffering PTSD. Testosterone's protective role on the pathogenic effect of low cortisol has already been hypothesized (Josephs et al., 2017) and is supported by rodent results demonstrating that exogenous testosterone reduces PTSD-like symptoms (Fenchel et al., 2015; Lynch et al., 2016).

In contrast to testosterone, plasma levels of estradiol were not affected by trauma. It remains to be established whether estradiol plays a role in PTSD symptomatology or whether the progesterone/ estradiol ratio may be essential for understating sex-dependent differences in fear and PTSD (Seligowski et al., 2020).

\section{Treatment Efficacy in Traumatized Male and Female Mice}

The present study's final goal was to examine whether treatment efficacy in a mouse PTSD-like model depended on the subjects' sex. Stress-induced freezing was reduced by pramipexole in both sexes, but tiagabine decreased it only in female mice.

\section{Sex-independent Efficacy of Pramipexole}

The clinical efficacy of pramipexole in PTSD has not yet been established (https://clinicaltrials.gov, ref. no NCT03765138, Research Foundation for Mental Hygiene,I, 2019). Figure 2 demonstrates that pramipexole reduced exaggerated freezing in traumatized female and male mice, suggesting its potential efficacy in PTSD treatment.

The rationale of using dopamine-mimetics in PTSD treatment is based on two hypotheses, implying faster fear memory extinction (Gerlicher et al., 2018) and facilitated stress adaptation (Cabib and Puglisi-Allegra, 2012; Douma and de Kloet, 2020). PTSD patients showed reduced cerebrospinal dopamine metabolites (Geracioti et al., 2013) and increased baseline dopamine beta-hydroxylase (Hamner and Gold, 1998), suggesting that dopamine-driven mechanisms are impaired after trauma.

Present study showing that $\mathrm{D}_{2}$-like type receptors agonist reduced conditioned fear but not hormonal response suggests that pramipexole did not affect stress adaptation, supporting inefficacy of quinpirole to reduce plasma corticosterone in rats subjected to electric shock stressor (De Oliveira et al., 2013) or selegiline in mice exposed to forced swim (Kasai et al., 2017).

Of note, pramipexole increased testosterone levels in nonstressed males. It agrees with findings by Okun et al. (2014), which shows elevated testosterone due to pramipexole in men and corresponds with the postulated testosterone's protective role in low corticosterone conditions (Josephs et al., 2017).

\section{Sex-dependent Efficacy of Tiagabine}

Tiagabine has already been tested in PTSD patients, and while studies reported ambiguous results (Connor et al., 2006; Davidson et al., 2007), the medication presented greater efficacy in women, agreeing with present data (Figure 2A).

PTSD patients demonstrate deficits related to GABA-ergic neurotransmission (Geuze et al., 2008; Trousselard et al., 2016; Rasmusson et al., 2019), although, in women, they are additionally magnified by specific blocks in progesterone metabolism resulting in an insufficient GABA-ergic neurosteroid modulation (Pineles et al., 2018; Rasmusson et al., 2019). Thus, the sex-specific efficacy of tiagabine may rely on complementing GABA-ergic neurotransmission, which is impaired in women.

\section{Study Limitations}

This study suffers from some limitations, including the use of isoflurane instead of diethyl ether as one of the mSPS components, which might have affected trauma efficacy (Knox et al., 2012). The use of isoflurane was due to the present ethical guidelines, but care was taken to anesthetize all mice efficiently. 
Second, between mSPS and the measurement of its effects, we repeatedly injected animals, though it is unlikely that the injections induced stress comparable with restraint or forced swim as in the rat study (Liberzon et al., 1999). Finally, in the present fear conditioning test, a solely contextual conditioned cue was applied as in the PTSD studies investigating traumaunrelated conditioned fear. This approach has already been used to examine PTSD-like phenotype of SPS-exposed rats (e.g., Takahashi et al., 2006; Kohda et al., 2007) and is supported by Orr et al. (2000) findings showing that following de novo conditioning, patients demonstrated increased fear evoked by conditional visual cues.

\section{CONCLUSION}

We report that plasma corticosterone depends on trauma and sex in mice suggesting sex-dependent HPA adaptation to severe stress purportedly biasing treatment outcome. Current results indicate the potential efficacy of $\mathrm{D}_{2}$-like receptor agonists in the treatment of PTSD in male and female subjects and of GABAmimetics in females.

\section{DATA AVAILABILITY STATEMENT}

The original contributions presented in the study are included in the article/Supplementary Material, further inquiries can be directed to the corresponding author.

\section{REFERENCES}

Aardal-Eriksson, E., Eriksson, T. E., and Thorell, L.-H. (2001). Salivary Cortisol, Posttraumatic Stress Symptoms, and General Health in the Acute Phase and during 9-month Follow-Up. Biol. Psychiatry 50, 986-993. doi:10.1016/s00063223(01)01253-7

American Psychiatric Association (2013). Diagnostic and Statistical Manual of Mental Disorders: DSM-5. 5th ed. Am. Psychiatr. Publ. Inc.

Cabib, S., and Puglisi-Allegra, S. (2012). The Mesoaccumbens Dopamine in Coping with Stress. Neurosci. Biobehavioral Rev. 36, 79-89. doi:10.1016/j.neubiorev. 2011.04.012

Cardinal, R. N., and Aitken, M. R. F. (2006). ANOVA for the Behavioral Sciences Researcher. 1st ed. New York: Abingdon, Oxon: Psychology Press . doi:10.4324/ 9780203763933

Carmi, L., Fostick, L., Burshtein, S., Cwikel-Hamzany, S., and Zohar, J. (2016). PTSD Treatment in Light of DSM-5 and the "golden Hours" Concept. CNS Spectr. 21, 279-282. doi:10.1017/S109285291600016X

Clark, J. W., Drummond, S. P. A., Hoyer, D., and Jacobson, L. H. (2019). Sex Differences in Mouse Models of Fear Inhibition: Fear Extinction, Safety Learning, and Fear-Safety Discrimination. Br. J. Pharmacol. 176, 4149-4158. doi:10.1111/bph.14600

Connor, K. M., Davidson, J. R. T., Weisler, R. H., Zhang, W., and Abraham, K. (2006). Tiagabine for Posttraumatic Stress Disorder: Effects of Open-Label and Double-Blind Discontinuation Treatment. Psychopharmacology 184, 21-25. doi:10.1007/s00213-005-0265-3

Danan, D., Matar, M. A., Kaplan, Z., Zohar, J., and Cohen, H. (2018). Blunted Basal Corticosterone Pulsatility Predicts post-exposure Susceptibility to PTSD Phenotype in Rats. Psychoneuroendocrinology 87, 35-42. doi:10.1016/j. psyneuen.2017.09.023

\section{ETHICS STATEMENT}

The animal study was reviewed and approved by 1st Local Ethics Committee of the Jagiellonian University in Kraków Medyczna 9 st. 30-688 Kraków Poland.

\section{AUTHOR CONTRIBUTIONS}

NM-R designed the experiments; NM-R and KS performed the behavioral experiments; NM-R and JG-A performed ELISA; NM$\mathrm{R}$ and $\mathrm{PP}$ performed data analysis; NM-R, PP and KS wrote the manuscript. All authors approved the manuscript.

\section{FUNDING}

This study was funded by the statutory funds K/ZDS/007869 of the Faculty of Pharmacy, K/ZDS/006281 of the Faculty of Health Sciences, Jagiellonian University Medical College, as well as the Statutory Activity of the Maj Institute of Pharmacology, Polish Academy of Sciences in Kraków.

\section{SUPPLEMENTARY MATERIAL}

The Supplementary Material for this article can be found online at: https://www.frontiersin.org/articles/10.3389/fphar.2021.691598/ full\#supplementary-material

Davidson, J. R. T., Brady, K., Mellman, T. A., Stein, M. B., and Pollack, M. H. (2007). The Efficacy and Tolerability of Tiagabine in Adult Patients with posttraumatic Stress Disorder. J. Clin. Psychopharmacol. 27, 85-88. doi:10.1097/ JCP.0b013e31802e5115

De Oliveira, A. R., Reimer, A. E., Reis, F. M. C. V., and Brandão, M. L. (2013). Conditioned Fear Response Is Modulated by a Combined Action of the Hypothalamic-Pituitary-Adrenal axis and Dopamine Activity in the Basolateral Amygdala. Eur. Neuropsychopharmacol. 23, 379-389. doi:10. 1016/j.euroneuro.2012.05.007

Dopfel, D., Perez, P. D., Verbitsky, A., Bravo-Rivera, H., Ma, Y., Quirk, G. J., et al. (2019). Individual Variability in Behavior and Functional Networks Predicts Vulnerability Using an Animal Model of PTSD. Nat. Commun. 10, 2372. doi:10. 1038/s41467-019-09926-Z

Douma, E. H., and de Kloet, E. R. (2020). Stress-induced Plasticity and Functioning of Ventral Tegmental Dopamine Neurons. Neurosci. Biobehavioral Rev. 108, 48-77. doi:10.1016/j.neubiorev.2019.10.015

Dunlop, B. W., and Wong, A. (2019). The Hypothalamic-Pituitary-Adrenal axis in PTSD: Pathophysiology and Treatment Interventions. Prog. NeuroPsychopharmacology Biol. Psychiatry 89, 361-379. doi:10.1016/j.pnpbp.2018.10.010

Fenchel, D., Levkovitz, Y., Vainer, E., Kaplan, Z., Zohar, J., and Cohen, H. (2015). Beyond the HPA-axis: The Role of the Gonadal Steroid Hormone Receptors in Modulating Stress-Related Responses in an Animal Model of PTSD. Eur. Neuropsychopharmacol. 25, 944-957. doi:10.1016/j.euroneuro.2015.02.004

Geracioti, T. D., Jefferson-Wilson, L., Strawn, J. R., Baker, D. G., Dashevsky, B. A., Horn, P. S., et al. (2013). Effect of Traumatic Imagery on Cerebrospinal Fluid Dopamine and Serotonin Metabolites in Posttraumatic Stress Disorder. J. Psychiatr. Res. 47, 995-998. doi:10.1016/j.jpsychires.2013.01.023

Gerlicher, A. M. V., Tüscher, O., and Kalisch, R. (2018). Dopamine-dependent Prefrontal Reactivations Explain Long-Term Benefit of Fear Extinction. Nat. Commun. 9, 4294. doi:10.1038/s41467-018-06785-y 
Geuze, E., Van Berckel, B. N. M., Lammertsma, A. A., Boellaard, R., De Kloet, C. S., Vermetten, E., et al. (2008). Reduced GABAA Benzodiazepine Receptor Binding in Veterans with post-traumatic Stress Disorder. Mol. Psychiatry 13, 74-83. doi:10.1038/sj.mp.4002054

Gill, J. M., Szanton, S. L., and Page, G. G. (2005). Biological Underpinnings of Health Alterations in Women with PTSD: A Sex Disparity. Biol. Res. Nurs. 7, 44-54. doi:10.1177/1099800405276709

Hamner, M. B., and Gold, P. B. (1998). Plasma Dopamine Beta-Hydroxylase Activity in Psychotic and Non-psychotic post-traumatic Stress Disorder. Psychiatry Res. 77, 175-181. doi:10.1016/s0165-1781(98)00002-x

Heck, A. L., and Handa, R. J. (2019). Sex Differences in the HypothalamicPituitary-Adrenal axis' Response to Stress: an Important Role for Gonadal Hormones. Neuropsychopharmacol 44, 45-58. doi:10.1038/s41386-0180167-9

Josephs, R. A., Cobb, A. R., Lancaster, C. L., Lee, H.-J., and Telch, M. J. (2017). Dual-hormone Stress Reactivity Predicts Downstream War-Zone StressEvoked PTSD. Psychoneuroendocrinology 78, 76-84. doi:10.1016/j.psyneuen. 2017.01.013

Karlović, D., Serretti, A., Marčinko, D., Martinac, M., Silić, A., and Katinić, K. (2012). Serum Testosterone Concentration in Combat-Related Chronic Posttraumatic Stress Disorder. Neuropsychobiology 65, 90-95. doi:10.1159/ 000329556

Kasai, S., Yoshihara, T., Lopatina, O., Ishihara, K., and Higashida, H. (2017). Selegiline Ameliorates Depression-like Behavior in Mice Lacking the CD157/ BST1 Gene, a Risk Factor for Parkinson's Disease. Front. Behav. Neurosci. 11, 75. doi:10.3389/fnbeh.2017.00075

Keller, S. M., Schreiber, W. B., Staib, J. M., and Knox, D. (2015). Sex Differences in the Single Prolonged Stress Model. Behav. Brain Res. 286, 29-32. doi:10.1016/j. bbr.2015.02.034

Kessler, R. C., Sonnega, A., Bromet, E., Hughes, M., and Nelson, C. B. (1995). Posttraumatic Stress Disorder in the National Comorbidity Survey. Arch. Gen. Psychiatry 52, 1048. doi:10.1001/archpsyc.1995.03950240066012

Kessler, R. (2003). Epidemiology of Women and Depression. J. Affective Disord. 74, 5-13. doi:10.1016/S0165-0327(02)00426-3

Kiss, B., Laszlovszky, I., Krámos, B., Visegrády, A., Bobok, A., Lévay, G., et al. (2021). Neuronal Dopamine D3 Receptors: Translational Implications for Preclinical Research and Cns Disorders. Biomolecules 11, 104-139. doi:10. 3390/biom11010104

Knox, D., Nault, T., Henderson, C., and Liberzon, I. (2012). Glucocorticoid Receptors and Extinction Retention Deficits in the Single Prolonged Stress Model. Neuroscience 223, 163-173. doi:10.1016/j.neuroscience.2012.07.047

Kohda, K., Harada, K., Kato, K., Hoshino, A., Motohashi, J., Yamaji, T., et al. (2007). Glucocorticoid Receptor Activation Is Involved in Producing Abnormal Phenotypes of Single-Prolonged Stress Rats: A Putative post-traumatic Stress Disorder Model. Neuroscience 148, 22-33. doi:10.1016/j.neuroscience.2007. 05.041

Leggio, G. M., Di Marco, R., Gulisano, W., D’Ascenzo, M., Torrisi, S. A., Geraci, F., et al. (2019). Dopaminergic-GABAergic Interplay and Alcohol Binge Drinking. Pharmacol. Res. 141, 384-391. doi:10.1016/j.phrs.2019.01.022

Leggio, G. M., Torrisi, S. A., Mastrogiacomo, R., Mauro, D., Chisari, M., Devroye, C., et al. (2021). The Epistatic Interaction between the Dopamine D3 Receptor and Dysbindin-1 Modulates Higher-Order Cognitive Functions in Mice and Humans. Mol. Psychiatry 26, 1272-1285. doi:10.1038/s41380-019-0511-4

Liberzon, I., Krstov, M., and Young, E. A. (1997). Stress-Restress: Effects on Acth and Fast Feedback. Psychoneuroendocrinology 22, 443-453. doi:10.1016/s03064530(97)00044-9

Liberzon, I., López, J. F., Flagel, S. B., Vázquez, D. M., and Young, E. A. (1999). Differential Regulation of Hippocampal Glucocorticoid Receptors mRNA and Fast Feedback: Relevance to Post-Traumatic Stress Disorder. J. Neuroendocrinology 11, 11-17. doi:10.1046/j.1365-2826.1999.00288.x

Lynch, J. F., Vanderhoof, T., Winiecki, P., Latsko, M. S., Riccio, D. C., and Jasnow, A. M. (2016). Aromatized Testosterone Attenuates Contextual Generalization of Fear in Male Rats. Horm. Behav. 84, 127-135. doi:10.1016/j.yhbeh.2016. 06.007

Malikowska, N., Sałat, K., and Popik, P. (2019b). Effects of Ketamine and Tiagabine in Mice Exposed to Single Prolonged Stress Protocol, a Mouse Model of Posttraumatic Stress Disorder. Eur. Neuropsychopharmacol. 29, S217-S218. doi:10.1016/j.euroneuro.2018.11.355
Malikowska-Racia, N., Sałat, K., Nowaczyk, A., Fijałkowski, Ł., and Popik, P. (2019a). Dopamine D2/D3 Receptor Agonists Attenuate PTSD-like Symptoms in Mice Exposed to Single Prolonged Stress. Neuropharmacology 155, 1-9. doi:10.1016/j.neuropharm.2019.05.012

Mental Hygiene, I(2019). NCT03765138: Combined Treatment of Prolonged Exposure and Pramipexole for Posttraumatic Stress Disorder and Depression. NCT03765138 Comb. Treat. Prolong. Expo. Pramipexole Posttraumatic Stress Disord. Depress, Available at: https://clinicaltrials.gov .

Metzger, L. J., Carson, M. A., Lasko, N. B., Paulus, L. A., Orr, S. P., Pitman, R. K., et al. (2008). Basal and Suppressed Salivary Cortisol in Female Vietnam Nurse Veterans with and without PTSD. Psychiatry Res. 161, 330-335. doi:10.1016/j. psychres.2008.04.020

Morey, R. A., Dunsmoor, J. E., Dunsmoor, J. E., Haswell, C. C., Brown, V. M., Vora, A., et al. (2015). Fear Learning Circuitry Is Biased toward Generalization of Fear Associations in Posttraumatic Stress Disorder. Transl Psychiatry 5, e700. doi:10. 1038/tp.2015.196

Morris, M. C., Hellman, N., Abelson, J. L., and Rao, U. (2016). Cortisol, Heart Rate, and Blood Pressure as Early Markers of PTSD Risk: A Systematic Review and Meta-Analysis. Clin. Psychol. Rev. 49, 79-91. doi:10.1016/j.cpr.2016.09.001

Nahvi, R. J., Nwokafor, C., Serova, L. I., and Sabban, E. L. (2019). Single Prolonged Stress as a Prospective Model for Posttraumatic Stress Disorder in Females. Front. Behav. Neurosci. 13, 17. doi:10.3389/fnbeh.2019.00017

Okun, M. S., Wu, S. S., Jennings, D., Marek, K., Rodriguez, R. L., and Fernandez, H. H. (2014). Testosterone Level and the Effect of Levodopa and Agonists in Early Parkinson Disease: Results from the INSPECT Cohort. J. Clin. Mov. Disord. 1, 8. doi:10.1186/2054-7072-1-8

Orr, S. P., Metzger, L. J., Lasko, N. B., Macklin, M. L., Peri, T., and Pitman, R. K. (2000). De Novo conditioning in Trauma-Exposed Individuals with and without Posttraumatic Stress Disorder. J. Abnormal Psychol. 109, 290-298. doi:10.1037/0021-843X.109.2.290

Perrine, S. A., Eagle, A. L., George, S. A., Mulo, K., Kohler, R. J., Gerard, J., et al. (2016). Severe, Multimodal Stress Exposure Induces PTSD-like Characteristics in a Mouse Model of Single Prolonged Stress. Behav. Brain Res. 303, 228-237. doi:10.1016/j.bbr.2016.01.056

Perugini, M., Gallucci, M., and Costantini, G. (2018). A Practical Primer to Power Analysis for Simple Experimental Designs. Int. Rev. Soc. Psychol. 31. doi:10. 5334/irsp.181

Pineles, S. L., Nillni, Y. I., Pinna, G., Irvine, J., Webb, A., Arditte Hall, K. A., et al. (2018). PTSD in Women Is Associated with a Block in Conversion of Progesterone to the GABAergic Neurosteroids Allopregnanolone and Pregnanolone Measured in Plasma. Psychoneuroendocrinology 93, 133-141. doi:10.1016/j.psyneuen.2018.04.024

Pooley, A. E., Benjamin, R. C., Sreedhar, S., Eagle, A. L., Robison, A. J., MazeiRobison, M. S., et al. (2018). Sex Differences in the Traumatic Stress Response: The Role of Adult Gonadal Hormones. Biol. Sex. Differ. 9, 32. doi:10.1186/ s13293-018-0192-8

Rasmusson, A. M., King, M. W., Valovski, I., Gregor, K., Scioli-Salter, E., Pineles, S. L., et al. (2019). Relationships between Cerebrospinal Fluid GABAergic Neurosteroid Levels and Symptom Severity in Men with PTSD. Psychoneuroendocrinology 102, 95-104. doi:10.1016/j.psyneuen.2018. 11.027

Rincón-Cortés, M., and Grace, A. A. (2017). Sex-dependent Effects of Stress on Immobility Behavior and VTA Dopamine Neuron Activity: Modulation by Ketamine. Int. J. Neuropsychopharmacol. 20, 823-832. doi:10.1093/ijnp/ pyx048

Salat, K., Podkowa, A., Mogilski, S., Zaręba, P., Kulig, K., Salat, R., et al. (2015). The Effect of GABA Transporter 1 (GAT1) Inhibitor, Tiagabine, on ScopolamineInduced Memory Impairments in Mice. Pharmacol. Rep. 67, 1155-1162.

Sarkar, J., Wakefield, S., MacKenzie, G., Moss, S. J., and Maguire, J. (2011). Neurosteroidogenesis Is Required for the Physiological Response to Stress: Role of Neurosteroid-Sensitive GABAA Receptors. J. Neurosci. 31, 18198-18210. doi:10.1523/JNEUROSCI.2560-11.2011

Seligowski, A. V., Hurly, J., Mellen, E., Ressler, K. J., and Ramikie, T. S. (2020). Translational Studies of Estradiol and Progesterone in Fear and PTSD. Eur. J. Psychotraumatology 11, 1723857. doi:10.1080/20008198.2020. 1723857

Sillivan, S. E., Joseph, N. F., Jamieson, S., King, M. L., Chévere-Torres, I., Fuentes, I., et al. (2017). Susceptibility and Resilience to Posttraumatic Stress Disorder-like 
Behaviors in Inbred Mice. Biol. Psychiatry 82, 924-933. doi:10.1016/j.biopsych. 2017.06.030

Speer, K. E., Semple, S., Naumovski, N., D'Cunha, N. M., and McKune, A. J. (2019). HPA axis Function and Diurnal Cortisol in post-traumatic Stress Disorder: A Systematic Review. Neurobiol. Stress 11, 100180. doi:10.1016/j.ynstr.2019. 100180

Takahashi, T., Morinobu, S., Iwamoto, Y., and Yamawaki, S. (2006). Effect of Paroxetine on Enhanced Contextual Fear Induced by Single Prolonged Stress in Rats. Psychopharmacology 189, 165-173. doi:10.1007/s00213-006-0545-6

Torrisi, S. A., Lavanco, G., Maurel, O. M., Gulisano, W., Laudani, S., Geraci, F., et al. (2021). A Novel Arousal-Based Individual Screening Reveals Susceptibility and Resilience to PTSD-like Phenotypes in Mice. Neurobiol. Stress 14, 100286. doi:10.1016/j.ynstr.2020.100286

Torrisi, S. A., Leggio, G. M., Drago, F., and Salomone, S. (2019). Therapeutic Challenges of Post-traumatic Stress Disorder: Focus on the Dopaminergic System. Front. Pharmacol. 10, 404. doi:10.3389/FPHAR.2019.00404

Trousselard, M., Lefebvre, B., Caillet, L., Andruetan, Y., de Montleau, F., Denis, J., et al. (2016). Is Plasma GABA Level a Biomarker of Post-Traumatic Stress Disorder (PTSD) Severity? A Preliminary Study. Psychiatry Res. 241, 273-279. doi:10.1016/j.psychres.2016.05.013

VanElzakker, M. B., Kathryn Dahlgren, M., Caroline Davis, F., Dubois, S., and Shin, L. M. (2014). From Pavlov to PTSD: The Extinction of Conditioned Fear in Rodents, Humans, and Anxiety Disorders. Neurobiol. Learn. Mem. 113, 3-18. doi:10.1016/j.nlm.2013.11.014
Verbitsky, A., Dopfel, D., and Zhang, N. (2020). Rodent Models of post-traumatic Stress Disorder: Behavioral Assessment. Transl Psychiatry 10, 132. doi:10.1038/ s41398-020-0806-x

Yehuda, R., Southwick, S. M., Krystal, J. H., Bremner, D., Charney, D. S., and Mason, J. W. (1993). Enhanced Suppression of Cortisol Following Dexamethasone Administration in Posttraumatic Stress Disorder. Am. J. Psychiatry 150, 83-86. doi:10.1176/ajp.150.1.83

Yehuda, R., Hoge, C. W., McFarlane, A. C., Vermetten, E., Lanius, R. A., Nievergelt, C. M., et al. (2015). Post-traumatic Stress Disorder. Nat. Rev. Dis. Primers 1, 15057. doi:10.1038/nrdp.2015.57

Yehuda, R. (2002). Post-traumatic Stress Disorder. N. Engl. J. Med. 346, 108-114. doi:10.1056/NEJMra012941

Conflict of Interest: The authors declare that the research was conducted in the absence of any commercial or financial relationships that could be construed as a potential conflict of interest.

Copyright $\odot 2021$ Malikowska-Racia, Salat, Gdula-Argasinska and Popik. This is an open-access article distributed under the terms of the Creative Commons Attribution License (CC BY). The use, distribution or reproduction in other forums is permitted, provided the original author(s) and the copyright owner(s) are credited and that the original publication in this journal is cited, in accordance with accepted academic practice. No use, distribution or reproduction is permitted which does not comply with these terms. 